\title{
Differentiating low and high grade mucoepidermoid carcinoma of the salivary glands using $\mathrm{CT}$ radiomics
}

\author{
Michael H. Zhang ${ }^{1}$, Adam Hasse ${ }^{2}$, Timothy Carroll ${ }^{2}$, Alexander T. Pearson ${ }^{3}$, Nicole A. Cipriani ${ }^{4}$, \\ Daniel T. Ginat ${ }^{5} \wedge$
}

${ }^{1}$ Pritzker School of Medicine, The University of Chicago, Chicago IL, USA; ${ }^{2}$ Graduate Program in Medical Physics, The University of Chicago, Chicago, IL, USA; ${ }^{3}$ Department of Medicine, The University of Chicago, Chicago IL, USA; ${ }^{4}$ Department of Pathology, The University of Chicago, Chicago IL, USA; ${ }^{5}$ Department of Radiology, The University of Chicago, Chicago IL, USA

Contributions: (I) Conception and design: MH Zhang, DT Ginat; (II) Administrative support: All authors; (III) Provision of study materials or patients: AT Pearson, NA Cipriani, DT Ginat; (IV) Collection and assembly of data: All authors; (V) Data analysis and interpretation: All authors; (VI) Manuscript writing: All authors; (VII) Final approval of manuscript: All authors.

Correspondence to: Daniel T. Ginat, MD, MS. University of Chicago, 5841 S Maryland Avenue, Chicago, IL 60637, USA. Email: dtg1@uchicago.edu.

\begin{abstract}
Background: The purpose of this study is to determine if Haralick texture analysis on CT imaging of mucoepidermoid carcinomas (MEC) can differentiate low-grade and high-grade tumors.

Methods: A retrospective review of 18 patients with MEC of the salivary glands, corresponding CT imaging and pathology report was performed. Tumors were manually segmented and image analysis was performed to calculate radiomic features. Radiomic features were compared between low-grade and highgrade MEC. A multivariable logistic regression model and receiver operating characteristic analysis was performed.
\end{abstract}

Results: A total of 18 patients (mean age, 51, range 9-83 years, 8 men and 10 women) were included. Nine patients had low-grade pathology and nine patients had high-grade pathology. Of the 18 cases, 7 (39\%) occurred in the parotid gland and 11 (61\%) occurred in minor salivary glands. No individual feature was significantly different between low-grade and high-grade MEC. A logistic regression model including surface regularity, energy and information measure II of correlation was performed and was able to predict high-grade MEC accurately (sensitivity 89\%, specificity 68\%). The area under the receiver operating characteristic curve was 0.802 .

Conclusions: High-grade MEC tend to have a low energy, high correlation texture as well as surface irregularity. Together, these three features may comprise a tumor phenotype that is able to predict highgrade pathology in MECs.

Keywords: Salivary gland; mucoepidermoid carcinoma (MEC); texture analysis; CT; grade

Submitted Nov 15, 2020. Accepted for publication Mar 12, 2021.

doi: $10.21037 /$ gs-20-830

View this article at: http://dx.doi.org/10.21037/gs-20-830

\section{Introduction}

Mucoepidermoid carcinoma (MEC) is a salivary gland neoplasm accounting for approximately $30 \%$ of salivary malignancies and consisting of mucus cells, intermediate cells and squamous cells (1). It has varying potential for aggressive behavior, characterized by tumor grade. The reported 5-year survival for MEC ranges from $92-100 \%$ for low grade tumors, $62-92 \%$ for intermediate grade, and $0-43 \%$ for high grade tumors (2). Treatment decisions for MEC are highly dependent on tumor grade, given their remarkable differences in clinical behavior. Low-grade

^ORCID: 0000-0003-3301-4364. 
MEC is traditionally treated with surgery whereas adjuvant radiation therapy is typically reserved for high-grade pathology $(3,4)$.

Radiomics is an ongoing field of research that enables radiologists to obtain additional information from standard medical images. These quantitative biomarkers include shape features such as tumor volume and surface regularity as well as voxel-based texture features (5). These features have been demonstrated to correlate with histopathological grade, molecular biomarkers and prognosis for various tumors, such as oropharyngeal, esophageal and breast cancer (6-10). For example, our group previously found correlations between tumor morphology and Ki67 and p53 expression in glioblastoma (6). Another study showed success using textural parameters on CT to differentiate histologic subtypes of peripheral bronchogenic carcinoma (11). Radiomics has also been an area of active research in the realm of head and neck cancers. Yu et al. was able to discriminate human papilloma virus (HPV) positive and HPV negative oropharyngeal cancers based contrast enhanced CT imaging (12). Another study showed the added value of radiomics in improving prediction of overall survival and progression-free survival in patients with locally advanced head and neck squamous cell carcinomas (HNSCC) (13).

While radiomic features were able to successfully discriminate between histologic grade in HNSCC tumors in addition to other histopathological features such as perineural invasion, lymphovascular invasion and extracapsular spread, this present study is the first to do so in salivary gland tumors $(14,15)$. In the realm of salivary gland tumors, Cheng et al. recently developed a prognostic model incorporating PET/CT radiomics for patients with salivary gland tumors (16). Radiomic features have also been utilized to discriminate between benign and malignant parotid gland tumors on MR imaging $(17,18)$. The present study analyzes CT-based radiomic features in salivary gland tumors.

Given the ability of these imaging biomarkers to predict these important clinical and histologic factors in different tumor types, radiomics may also be able to provide noninvasive analysis of MEC and allow for more effective evaluation of tumor aggressiveness. A noninvasive quantitative analysis of these radiomic features could be clinically useful, particularly in cases where biopsies are difficult to obtain. This kind of radiomic analysis can also help assess tumor heterogeneity that may not be captured by biopsy. The purpose of our study was to evaluate the usefulness of radiomic biomarkers obtained from CT imaging to predict histopathological tumor grade of MEC. We present the following article in accordance with the STARD reporting checklist (available at http://dx.doi. org/10.21037/gs-20-830).

\section{Methods}

\section{Case selection}

The study was conducted in accordance with the Declaration of Helsinki (as revised in 2013). The study was approved by the institutional review board of the University of Chicago (IORG0000201) and individual consent for this retrospective analysis was waived. A retrospective review of 53 consecutive patients with low or high grade mucoepidermoid tumors treated at the University of Chicago from 1995 to 2017 was performed. Patients were excluded if relevant CT imaging or pathology report were not available. Patients were also excluded if no tumor mass was discernable on CT imaging. Both original and recurrent cancers were included. CT images were de-identified and imported to image analysis software.

\section{Pathologic analysis}

Diagnostic hematoxylin-eosin stain slides were graded according to the Brandwein grading system (19). In light of behavioral similarity between Brandwein low and intermediate grade tumors, they were grouped together as low grade for the purposes of this study (13).

\section{Image acquisition}

The neck CT scans were performed with intravenous nonionic iodinated contrast $(350 \mathrm{mg}$ of iodine per milliliter, Omnipaque) injected at a rate of $1.2 \mathrm{~mL} / \mathrm{s}$ and $55 \mathrm{~s}$ delay after the start of the injection. The scan parameters included $120 \mathrm{kV} ; 250 \mathrm{mAs}$; rotation time, 1.0 second; pitch, 0.75 ; collimation, $24 \mathrm{~mm} \times 1.2 \mathrm{~mm}$; section thickness, $3 \mathrm{~mm}$; intervals, $3 \mathrm{~mm}$, and display field of view, 20 to $25 \mathrm{~cm}$. A B30s smoothing algorithm was implemented without iterative reconstruction techniques.

\section{Tumor shape analysis}

The soft tissue window images were analyzed without further processing in MATLAB using in-house developed 


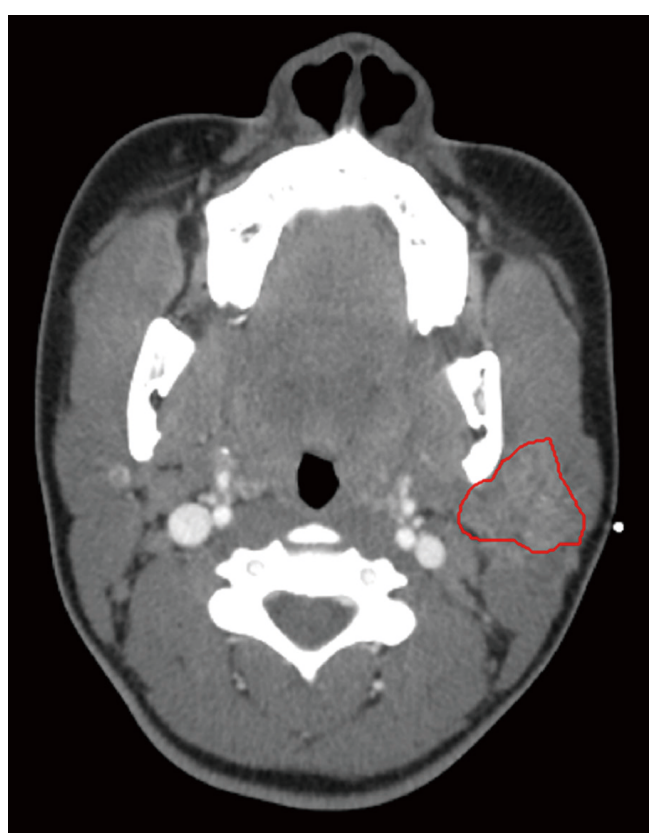

Figure 1 Axial image on contrast-enhanced computed tomography (CT) shows a left parotid gland mucoepidermoid carcinoma with a manually segmented region of interest (red outline).

software on MATLAB. The regions of interest for all the cases were obtained manually drawn by a single trained operator (MHZ) under the supervision of a board-certified neuroradiologist (Figure 1). The ROI was drawn on all axial slices where tumor could be visualized. The operator was blinded to pathological grade during segmentation. A 3D volume was generated from the axial segmentations and smoothed along the axial direction. Radiomic features were calculated for each lesion in MATLAB on the base image without noise correction, normalization or image transformations. The following features were calculated: surface regularity, mean intensity and standard deviation of intensity of the tumor, energy, contrast, variance, homogeneity, sum average, sum variance, sum entropy, entropy, difference variance, difference entropy, mean correlation, information measure of correlations and maximum correlation coefficient (20). Intensity was measured by Hounsfield units.

$$
\text { Surface regularity }=6 \sqrt{\pi} \frac{\text { Tumor Volume }}{(\text { Tumor Surface Area })^{3}}
$$

Haralick texture features are a set of gray-level cooccurrence matrix (GLCM) texture features. These features characterize texture based on the intensity of voxels (in Hounsfield units) and their specific spatial relationship to each other. The algorithms for these features were implemented as described by Haralick et al. and have been previously reviewed by the Imaging Biomarker Standardisation Initiative (20-22).

\section{Statistical analysis}

Texture features were evaluated for normality by the Shapiro-Wilk test and $\mathrm{P}$ values were calculated using Student's $t$-test or Wilcoxon rank-sum as appropriate. A threshold of $\mathrm{P}<0.05$ was considered statistically significant. A multivariable logistic regression model was developed to predict high-grade pathology from texture features. Three features were chosen from the model by ascending $\mathrm{P}$ value. Colinearity in the model was assessed to avoid redundancy in the model. Any variable with significant correlation with another independent variable, defined as $\left|R^{2}\right|>0.9$, was removed from the model. Multivariable regression was performed on surface regularity, mean energy and information measure of correlation II. An associated receiver operating characteristic (ROC) curve was developed by varying the threshold for predicted probability of high-grade pathology. All statistical analyses were performed with R, Version 3.6.1 (www.r-project.org).

\section{Results}

Of the 53 patients reviewed, a cohort of 18 patients was ultimately included (Figure 2). Patient characteristics are in Table 1. The mean age was 51 (range, 9-83 years). Ten patients (56\%) were female and eight patients (44\%) were male. Of the 18 patients, nine patients $(50 \%)$ had highgrade pathology and nine patients $(50 \%)$ had low-grade pathology. Seven cases were located in the parotid gland. Eleven cases occurred in a minor salivary gland (3 palate, 4 tongue, 1 floor of mouth, 1 mandible, 1 tonsillar and 1 retromolar trigone). Four tumors were recurrences.

Texture features of low-grade and high-grade MEC are reported in Table 2. No individual radiomic features were significantly different. High-grade MEC trended towards having lower energy $(0.50 \pm 0.04$ vs. $0.55 \pm 0.05, \mathrm{P}=0.06)$ and higher information measure II of correlation $(0.83 \pm 0.04 \mathrm{vs}$. $0.77 \pm 0.07, \mathrm{P}=0.08)$.

Radiomic features were selected for our multivariable logistic regression model to predict MEC grade. The features with the smallest $\mathrm{P}$ values in ascending order were energy $(\mathrm{P}=0.06)$, information measure of correlation II $(\mathrm{P}=0.08)$, sum entropy $(\mathrm{P}=0.12)$, maximum correlation 


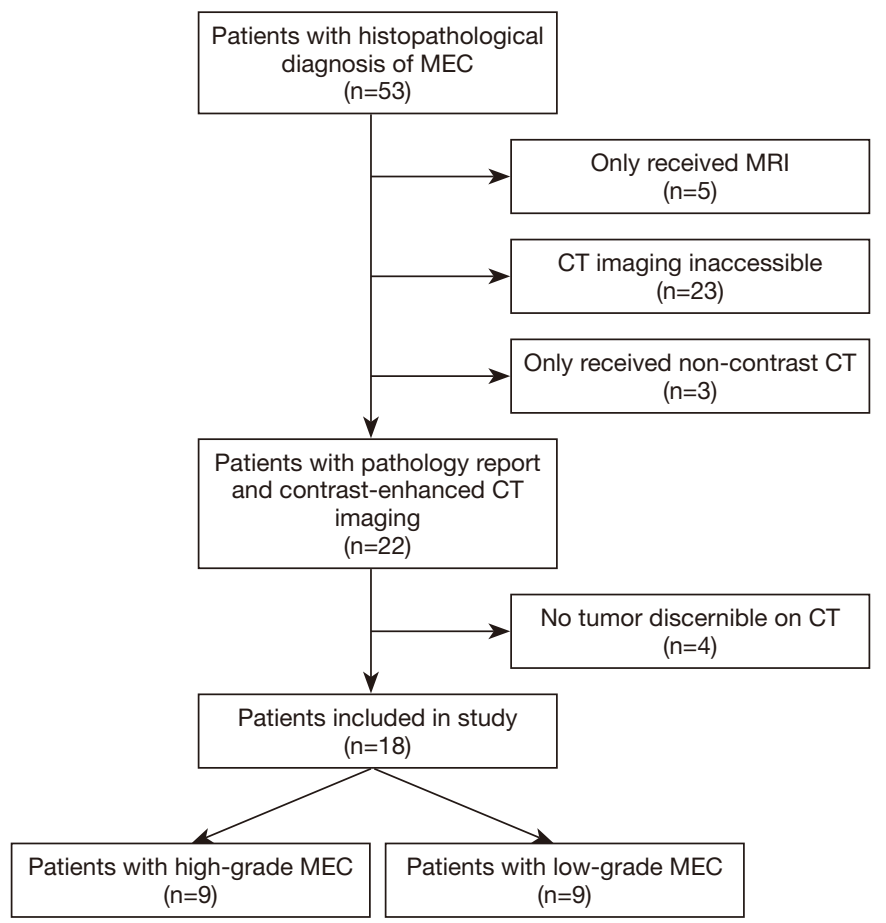

Figure 2 Flow chart of inclusion criteria.

Table 1 Clinical characteristics of the patient population

\begin{tabular}{|c|c|c|c|c|}
\hline Characteristic & All Patients & Low-grade & High-grade & $P$ value \\
\hline Age (years) & $51 \pm 21$ & $44 \pm 23$ & $56 \pm 19$ & 0.27 \\
\hline Sex & & & & 0.64 \\
\hline Female & $10(56 \%)$ & $4(44 \%)$ & $6(67 \%)$ & \\
\hline Tumor location & & & & 1.00 \\
\hline Parotid & 7 (39\%) & 3 (33\%) & $4(44 \%)$ & \\
\hline Minor & $11(61 \%)$ & $6(67 \%)$ & 5 (56\%) & \\
\hline Tongue & $4(22 \%)$ & $3(33 \%)$ & $1(11 \%)$ & \\
\hline Mandible & $1(6 \%)$ & $1(11 \%)$ & $0(0 \%)$ & \\
\hline Tonsillar & $1(6 \%)$ & $1(11 \%)$ & $0(0 \%)$ & \\
\hline Retromolar trigone & $1(6 \%)$ & $0(0 \%)$ & $1(11 \%)$ & \\
\hline Recurrent tumor & $4(22 \%)$ & $0(0 \%)$ & $4(44 \%)$ & 0.02 \\
\hline
\end{tabular}

Numerical data are mean \pm standard deviation. 
Table 2 Radiomic and Haralick texture features compared between low-grade and high-grade mucoepidermoid carcinoma

\begin{tabular}{|c|c|c|c|}
\hline Image feature & Low-grade & High-grade & $P$ value \\
\hline Intensity & $89 \pm 46$ & $79 \pm 37$ & 0.65 \\
\hline Standard deviation of intensity & $34 \pm 19$ & $48 \pm 48$ & 0.41 \\
\hline Energy & $0.55 \pm 0.05$ & $0.50 \pm 0.04$ & 0.06 \\
\hline Mean correlation & $0.81 \pm 0.09$ & $0.86 \pm 0.06$ & 0.19 \\
\hline Variance & $41 \pm 11$ & $38 \pm 7$ & 0.30 \\
\hline Homogeneity & $0.93 \pm 0.03$ & $0.94 \pm 0.02$ & 0.48 \\
\hline Sum average & $11 \pm 3$ & $10 \pm 2$ & 0.58 \\
\hline Entropy & $1.16 \pm 0.11$ & $1.23 \pm 0.11$ & 0.24 \\
\hline Difference variance & $3.3 \pm 1.3$ & $2.8 \pm 1.2$ & 0.48 \\
\hline Difference entropy & $0.24 \pm 0.07$ & $0.22 \pm 0.07$ & 0.50 \\
\hline Information measure I of correlation & $-0.58 \pm 0.13$ & $-0.66 \pm 0.11$ & 0.20 \\
\hline Information measure II of correlation & $0.77 \pm 0.07$ & $0.83 \pm 0.04$ & 0.08 \\
\hline Maximum correlation coefficient & $0.81 \pm 0.09$ & $0.86 \pm 0.06$ & 0.14 \\
\hline
\end{tabular}

Data are mean \pm standard deviation.

Table 3 Multivariable logistic regression analysis to determine high-grade mucoepidermoid carcinoma

\begin{tabular}{lcc}
\hline Image feature & Coefficient & P value \\
\hline Surface regularity & -2.374 & 0.81 \\
Energy & -14.137 & 0.33 \\
Information measure II of correlation & 13.254 & 0.17 \\
\hline
\end{tabular}

coefficient $(\mathrm{P}=0.14)$, mean correlation $(\mathrm{P}=0.19)$ and surface regularity $(\mathrm{P}=0.19)$. Next, features were assessed for collinearity. Energy was highly colinear with sum entropy $\left(\mathrm{R}^{2}=-0.93\right)$ so sum entropy was removed from the model. Information measure II of correlation was highly correlated to correlation (R2 $=0.97)$, information measure I of correlation $(\mathrm{R} 2=-0.94)$ and the maximum correlation coefficient $(\mathrm{R} 2=0.97)$. Therefore, only one correlation feature was used for our model (information measure of correlation II, chosen by ascending $\mathrm{P}$ value). Surface regularity was not significantly colinear with any other feature.
A multivariable logistic regression to predict Brandwein pathology was developed using information measure II of correlation, energy, and surface regularity (Table 3). At a threshold of 0.38 , the sensitivity was $89 \%$ and the specificity was $67 \%$ with an overall accuracy of $78 \%$. The confusion matrix is presented in Table 4. ROC analysis yielded an area under the curve (AUC) of 0.802 (Figure 3).

\section{Discussion}

The field of radiomics offers the ability to provide additional non-invasive information in the context of head and neck cancer imaging. CT-based radiomic features have been used to evaluate histopathological features such as HPV status, correlation with driver gene mutations and extranodal metastasis (23-26). Other studies demonstrated the ability of radiomics to provide additional insight regarding tumor response and clinical prognosis $(7,27,28)$. In this study we link a group of novel CT features to the pathological grade in MEC.

The results of this study show that a model of 
Table 4 Confusion matrix showing the multivariable model performance in predicting pathological grade

\begin{tabular}{lccc}
\hline Variable & Predicted: low-grade & Predicted: high-grade & Total \\
\hline Observed: low-grade & 6 & 3 & 9 \\
Observed: high-grade & 1 & 8 & 9 \\
Total & 7 & 11 & 18 \\
\hline
\end{tabular}

$n=18$.

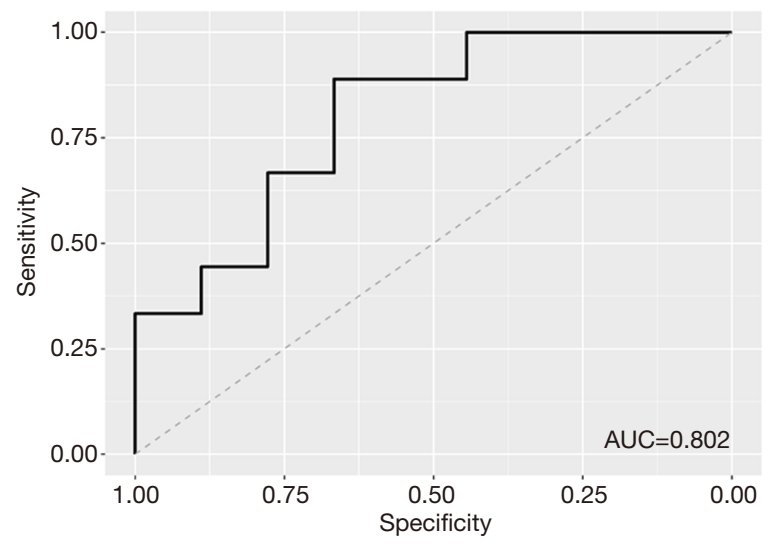

Figure 3 Receiver operating curve demonstrating performance of radiomic feature model for predicting mucoepidermoid carcinoma grade.

surface regularity and two GLCM features (energy, and information measure II of correlation) in contrast-enhanced CT imaging may accurately and noninvasively predict highgrade MEC. Although there are numerous studies that have linked radiomic features to histopathology, prognosis, and treatment response for a variety of other tumor types $(12,29,30)$, no previous studies have characterized the radiomic features of MEC. However, CT texture analysis has revealed tumor heterogeneity differences between pleomorphic adenoma and Warthin tumor (31).

Surface regularity is a radiomic feature comparing surface area to surface volume. Lower surface regularity represents a more complex tumor and spiculation (32). Yu et al. found that tumors with higher surface regularity and smaller diameters were more likely to be HPV positive and therefore associated with improved survival ( $\mathrm{AUC}=0.87$ and AUC $=0.92$ on two different datasets) (12). In our current study, we find similar results where high surface regularity correlated with less aggressive low-grade pathology. Similarly, low surface regularity has also been previously described to correlate with poor survival in cancers of other organ systems, including glioblastoma multiforme and hepatocellular carcinoma (30,33). Furthermore, surface regularity as a biomarker also has strong biological plausibility. In a study of 126 patients with HNSCC, poor surface regularity has been associated with suppression of numerous immune system pathways used in tumor surveillance including B-cell and T-cell signaling as well as cell adhesion pathways and cell architecture pathways (29).

Another feature of our model is energy, a GLCM texture feature which describes the uniformity of the image. In a radiomic-genomic study of patients with HNSCC, low energy was associated with elevated transcriptional activity of ribosomes and tumor protein synthesis (29). Likewise, energy has been used to characterize lung cancer and prostate cancer $(32,34)$. On CT imaging, Zayed et al. demonstrated significantly lower energy in lungs with a cancerous lesion, when compared to the fellow lung (34). Wibmer et al. showed lower energy in cancer lesions compared to noncancerous tissue on prostate, though this was calculated on MRI ADC mapping (35). Among the cases of prostate cancer, higher Gleason scores were associated with significantly lower mean energy. In our present study, we see a similar trend of lower energy in high-grade MEC.

Lastly, correlation measures if the grey level of a pixel is linearly dependent on its neighbors (20). In other words, correlation assesses for lines or linear features in an image (a homogenous, completely uniform image has a correlation of 0). Correlation may capture intratumoral vessels or possibly help characterize a heterogenous tumor. It is recognized that a tumor can have variations in genomic subtypes, growth factor and angiogenic factors as well as the tumor microenvironment (36). Heterogeneity is a key feature correlated with adverse tumor biology and poor prognosis (36).

Previously, correlation has been useful in characterizing tumor heterogeneity in non-small cell lung cancer (NSCLC). This model characterized tumor phenotype and predicted prognosis (7). Their radiomic model was 
then validated on patients with NSCLC and HNSCC, outperforming tumor volume and traditional TNM classification (7). By developing a model on NSCLC tumors and validating it on HNSCC, their study suggests radiomic features can reveal a tumor phenotype that is not organ specific yet prognostically valuable and complementary to traditional prognostication systems (7). Likewise, our model uses correlation as part of a three feature model to predict tumor grade. A previous study on MEC in the lung noted heterogenous tumor density on CT in $27 \%$ of low-grade MEC and $100 \%$ of high-grade MEC (37). It possible that correlation is capturing similar variations in tumor density on a smaller scale.

One strength of this study is the use of only three features in the model. Limiting the number of features avoids overfitting. Furthermore, radiomic features can be sensitive to image acquisition and reconstruction protocols $(5,38,39)$. The variety of CT scanners used to acquire our images adds a layer of robustness to our model. However, there are also limitations in our study. First, MEC is a relatively rare tumor and our study is limited by the small number of samples in our dataset. Also, only a single operator placed ROIs for the tumor and therefore the results may not be as generalizable. Another limitation was that internal validation was not possible given the number of patients. Future studies should validate these radiomic features on larger, external datasets. Ultimately, CT-based radiomics could help predict grade in difficult to biopsy cases. Further work may also lead to the use of the radiomic features identified in this study to better predict response to treatment or overall prognosis.

\section{Conclusions}

A group of CT texture features are able to predict pathological grade of MEC lesions with a high sensitivity and specificity. Our preliminary study demonstrates that high-grade MEC has a low surface regularity, low energy and high correlation phenotype that is distinguishable from low-grade MEC. This study serves as a proof of concept for using radiomic features as predictors of pathological grade in MEC.

\section{Acknowledgments}

Funding: None.

\section{Footnote}

Reporting Checklist: The authors have completed the STARD reporting checklist. Available at http://dx.doi.org/10.21037/ gs-20-830

Data Sharing Statement: Available at http://dx.doi. org/10.21037/gs-20-830

Peer Review File: Available at http://dx.doi.org/10.21037/gs20-830

Conflicts of Interest: All authors have completed the ICMJE uniform disclosure form (available at http://dx.doi. org/10.21037/gs-20-830). The authors have no conflicts of interest to declare.

Ethical Statement: The authors are accountable for all aspects of the work in ensuring that questions related to the accuracy or integrity of any part of the work are appropriately investigated and resolved. The study was conducted in accordance with the Declaration of Helsinki (as revised in 2013). The study was approved by the institutional review board of the University of Chicago (IORG0000201) and individual consent for this retrospective analysis was waived.

Open Access Statement: This is an Open Access article distributed in accordance with the Creative Commons Attribution-NonCommercial-NoDerivs 4.0 International License (CC BY-NC-ND 4.0), which permits the noncommercial replication and distribution of the article with the strict proviso that no changes or edits are made and the original work is properly cited (including links to both the formal publication through the relevant DOI and the license). See: https://creativecommons.org/licenses/by-nc-nd/4.0/.

\section{References}

1. Devaney K. Atlas of Tumor Pathology. Tumors of the Salivary Glands. Am J Surg Pathol 1997;21:361.

2. Seethala RR. An Update on Grading of Salivary Gland Carcinomas. Head Neck Pathol 2009;3:69-77.

3. Nance MA, Seethala RR, Wang Y, et al. Treatment and Survival Outcomes Based on Histologic Grading in Patients With Head and Neck Mucoepidermoid Carcinoma. Cancer 2008;113:2082-9. 
4. Spellman J, Calzada G. Mucoepidermoid Carcinoma: A 23-Year Experience with Emphasis on Low-Grade Tumors with Close/Positive Margins. Otolaryngol Head Neck Surg 2018;158:889-95.

5. Rizzo S, Botta F, Raimondi S, et al. Radiomics: the facts and the challenges of image analysis. Eur Radiol Exp 2018;2:36.

6. Hasse A, Dapash M, Jeong Y, et al. Correlation of postcontrast T1-weighted MRI surface regularity, tumor bulk, and necrotic volume with Ki67 and p53 in glioblastomas. Neuroradiology 2019;61:861-7.

7. Aerts HJWL, Velazquez ER, Leijenaar RTH, et al. Decoding tumour phenotype by noninvasive imaging using a quantitative radiomics approach. Nat Commun 2014;5:4006.

8. Yip C, Landau D, Kozarski R, et al. Primary esophageal cancer: heterogeneity as potential prognostic biomarker in patients treated with definitive chemotherapy and radiation therapy. Radiology 2014;270:141-8.

9. Pickles MD, Lowry M, Gibbs P. Pretreatment Prognostic Value of Dynamic Contrast-Enhanced Magnetic Resonance Imaging Vascular, Texture, Shape, and Size Parameters Compared With Traditional Survival Indicators Obtained From Locally Advanced Breast Cancer Patients. Invest Radiol 2016;51:177-85.

10. Leijenaar RTH, Carvalho S, Hoebers FJP, et al. External validation of a prognostic CT-based radiomic signature in oropharyngeal squamous cell carcinoma. Acta Oncol 2015;54:1423-9.

11. Kido S, Kuriyama K, Higashiyama M, et al. Fractal analysis of internal and peripheral textures of small peripheral bronchogenic carcinomas in thin-section computed tomography: comparison of bronchioloalveolar cell carcinomas with nonbronchioloalveolar cell carcinomas. J Comput Assist Tomogr 2003;27:56-61.

12. Yu K, Zhang Y, Yu Y, et al. Radiomic analysis in prediction of Human Papilloma Virus status. Clin Transl Radiat Oncol 2017;7:49-54.

13. Ou D, Blanchard P, Rosellini S, et al. Predictive and prognostic value of CT based radiomics signature in locally advanced head and neck cancers patients treated with concurrent chemoradiotherapy or bioradiotherapy and its added value to Human Papillomavirus status. Oral Oncology 2017;71:150-5.

14. Mukherjee P, Cintra M, Huang C, et al. CT-based Radiomic Signatures for Predicting Histopathologic Features in Head and Neck Squamous Cell Carcinoma. Radiology 2020;2:e190039.
15. Wu W, Ye J, Wang Q, et al. CT-Based Radiomics Signature for the Preoperative Discrimination Between Head and Neck Squamous Cell Carcinoma Grades. Front Oncol 2019;9:821.

16. Cheng NM, Hsieh CE, Fang YHD, et al. Development and validation of a prognostic model incorporating [18F] FDG PET/CT radiomics for patients with minor salivary gland carcinoma. EJNMMI Res 2020;10:74.

17. Gabelloni M, Faggioni L, Attanasio S, et al. Can Magnetic Resonance Radiomics Analysis Discriminate Parotid Gland Tumors? A Pilot Study. Diagnostics (Basel) 2020;10:900.

18. Shao S, Mao N, Liu W, et al. Epithelial salivary gland tumors: Utility of radiomics analysis based on diffusionweighted imaging for differentiation of benign from malignant tumors. J Xray Sci Technol 2020;28:799-808.

19. Cipriani NA, Lusardi JJ, McElherne J, et al. Mucoepidermoid Carcinoma: A Comparison of Histologic Grading Systems and Relationship to MAML2 Rearrangement and Prognosis. Am J Surg Pathol 2019;43:885-97.

20. Haralick RM, Shanmugam K, Dinstein I. Textural Features for Image Classification. IEEE Transactions on Systems, Man, and Cybernetics 1973;SMC-3(6):610-21.

21. Zwanenburg A, Vallières $M$, Abdalah MA, et al. The Image Biomarker Standardization Initiative: Standardized Quantitative Radiomics for High-Throughput Imagebased Phenotyping. Radiology 2020;295:328-38.

22. Image features - IBSI 0.0.1 dev documentation [Internet]. [cited 2021 Mar 5]. Available online: https://ibsi. readthedocs.io/en/latest/03_Image_features.html

23. Buch K, Fujita A, Li B, et al. Using Texture Analysis to Determine Human Papillomavirus Status of Oropharyngeal Squamous Cell Carcinomas on CT. AJNR Am J Neuroradiol 2015;36:1343-8.

24. Fujita A, Buch K, Li B, et al. Difference Between HPVPositive and HPV-Negative Non-Oropharyngeal Head and Neck Cancer: Texture Analysis Features on CT. J Comput Assist Tomogr 2016;40:43-7.

25. Zwirner K, Hilke FJ, Demidov G, et al. Radiogenomics in head and neck cancer: correlation of radiomic heterogeneity and somatic mutations in TP53, FAT1 and KMT2D. Strahlenther Onkol 2019;195:771-9.

26. Kann BH, Aneja S, Loganadane GV, et al. Pretreatment Identification of Head and Neck Cancer Nodal Metastasis and Extranodal Extension Using Deep Learning Neural Networks. Sci Rep 2018;8:14036.

27. Zhai T-T, Langendijk JA, van Dijk LV, et al. The prognostic value of CT-based image-biomarkers for head 
and neck cancer patients treated with definitive (chemo-) radiation. Oral Oncol 2019;95:178-86.

28. Elhalawani H, Kanwar A, Mohamed ASR, et al. Investigation of radiomic signatures for local recurrence using primary tumor texture analysis in oropharyngeal head and neck cancer patients. Sci Rep 2018;8:1524.

29. Zhu Y, Mohamed ASR, Lai SY, et al. Imaging-Genomic Study of Head and Neck Squamous Cell Carcinoma: Associations Between Radiomic Phenotypes and Genomic Mechanisms via Integration of The Cancer Genome Atlas and The Cancer Imaging Archive. JCO Clinical Cancer Informatics 2019;(3):1-9.

30. Kim J, Choi SJ, Lee SH, et al. Predicting Survival Using Pretreatment CT for Patients With Hepatocellular Carcinoma Treated With Transarterial Chemoembolization: Comparison of Models Using Radiomics. AJR Am J Roentgenol 2018;211:1026-34.

31. Jung YJ, Han M, Ha EJ, et al. Differentiation of salivary gland tumors through tumor heterogeneity: a comparison between pleomorphic adenoma and Warthin tumor using CT texture analysis. Neuroradiology 2020;62:1451-8.

32. Limkin EJ, Reuzé S, Carré A, et al. The complexity of tumor shape, spiculatedness, correlates with tumor radiomic shape features. Sci Rep 2019;9:4329.

33. Pérez-Beteta J, Molina-García D, Ortiz-Alhambra JA, et al. Tumor Surface Regularity at MR Imaging Predicts

Cite this article as: Zhang $\mathrm{MH}$, Hasse $\mathrm{A}$, Carroll $\mathrm{T}$, Pearson AT, Cipriani NA, Ginat DT. Differentiating low and high grade mucoepidermoid carcinoma of the salivary glands using CT radiomics. Gland Surg 2021;10(5):1646-1654. doi: $10.21037 /$ gs-20-830
Survival and Response to Surgery in Patients with Glioblastoma. Radiology 2018;288:218-25.

34. Zayed N, Elnemr HA. Statistical Analysis of Haralick Texture Features to Discriminate Lung Abnormalities. Int J Biomed Imaging 2015;2015:267807.

35. Wibmer A, Hricak H, Gondo T, et al. Haralick texture analysis of prostate MRI: utility for differentiating noncancerous prostate from prostate cancer and differentiating prostate cancers with different Gleason scores. Eur Radiol 2015;25:2840-50.

36. Ganeshan B, Miles KA. Quantifying tumour heterogeneity with CT. Cancer Imaging 2013;13:140-9.

37. Wang YQ, Mo YX, Li S, et al. Low-Grade and HighGrade Mucoepidermoid Carcinoma of the Lung: CT Findings and Clinical Features of 17 Cases. AJR Am J Roentgenol 2015;205:1160-6.

38. Solomon J, Mileto A, Nelson RC, et al. Quantitative Features of Liver Lesions, Lung Nodules, and Renal Stones at Multi-Detector Row CT Examinations: Dependency on Radiation Dose and Reconstruction Algorithm. Radiology 2016;279:185-94.

39. Meyer M, Ronald J, Vernuccio F, et al. Reproducibility of CT Radiomic Features within the Same Patient: Influence of Radiation Dose and CT Reconstruction Settings. Radiology 2019;293:583-91. 